\title{
miR-34a and miR-125a-5p inhibit proliferation and metastasis but induce apoptosis in hepatocellular carcinoma cells via repressing the MACC1- mediated PI3K/AKT/mTOR pathway
}

\author{
Y. M. ZHANG', Q. M. WU², L. Y. CHANG ${ }^{3}$, J. C. LIU $^{4, *}$ \\ ${ }^{1}$ General Medicine Department, Yantaishan Hospital, Yantai, Shandong 264001, China; ${ }^{2}$ Health Management Center, Qingdao Central Hospi- \\ tal, Qingdao, Shandong 266042, China; ${ }^{3}$ Health Management Center, Qingdao Cancer Hospital, Qingdao, Shandong 266042, China; ${ }^{4}$ Depart- \\ ment of Gastroenterology, Linyi People’s Hospital, Linyi, Shandong 251500, China
}

*Correspondence: bengpadao1j2@126.com

Received October 19, 2019 / Accepted January 7, 2020

\begin{abstract}
microRNA-34a (miR-34a) and microRNA-1251-5p (miR-125a-5p) were considered as tumor suppressors in hepatocellular carcinoma (HCC). Nevertheless, the modulatory mechanisms of miR-34a and miR-125a-5p in HCC haven't been completely understood. The levels of metastasis-associated with colon cancer 1 (MACC1) and miRNAs (miR-34a and miR-125a-5p) were determined by quantitative real-time polymerase chain reaction (qRT-PCR), and the levels of associated proteins were detected by western blot assay. Cell proliferation and metastasis were examined via Cell Counting Kit- 8 (CCK-8) and transwell assays, respectively. Cell apoptosis was measured through flow cytometry. The effect of MACC1 on HCC in vivo was explored via xenograft assay. Dual-luciferase reporter assay and RNA Immunoprecipitation (RIP) assay were implemented to explore the target correlation. The expression of MACC1 was upregulated in HCC tissues and cells. Knockdown of MACC1 inhibited proliferation and metastasis but expedited apoptosis of HCC cells and the repression of tumor growth in vivo was evoked by MACC1 downregulation. Both miR-34a and miR-125a-5p directly targeted MACC1 and repressed the expression of MACC1 in HCC cells. Overexpression of miR-34a or miR-125a-5p restrained cell proliferation and metastasis while induced apoptosis by downregulating MACC1 in HCC cells. miR-34a and miR-125a-5p repressed phosphoinositide 3-kinase (PI3K)/protein kinase B (AKT)/mammalian target of rapamycin (mTOR) signal pathway via reducing MACC1 in HCC cells. miR-34a and miR-125a-5p refrained proliferation and metastasis while motivated apoptosis in HCC cells through the PI3K/AKT/mTOR pathway by repressing MACC1. miR-34a and miR-125a-5p might be splendid biomarkers in the therapeutic strategies for HCC.
\end{abstract}

Key words: hepatocellular carcinoma, miR-34a, miR-125a-5p, MACC1, PI3K/AKT/mTOR pathway

Hepatocellular carcinoma (HCC) is a type of the most frequent malignancies and the second dominating cause of cancer-resulting in death worldwide [1]. The strongest risk factor for HCC is cirrhosis, along with cirrhosis-related hepatitis $\mathrm{B} / \mathrm{C}$ virus $(\mathrm{HBV} / \mathrm{HCV})$ infection $[2,3]$. The survival rates and prognosis remain quite scanty despite the great advancement of treatments, such as surgical excision, liver transplantation, microwave ablation, and interventional radiology [4-6]. Therefore, seeking more therapeutic targets for HCC becomes extremely imperative.

MicroRNAs (miRNAs) are involved in multifarious cellular processes by combining with the 3'-untranslated regions (3'-UTRs) of the downstream genes, bringing about the degradation of mRNA or the inhibition of translation at the post-transcriptional level [7, 8]. Increasing studies presented that different miRNAs were conductive to the HCC progression $[9,10]$. For instance, miR-27a and miR-18b served as available biomarkers in the prediction of HCV-associated HCC [11]; Wang et al. attested that miR-206 reduced cell proliferation and migration while accelerated apoptosis via inhibiting c-mesenchymal-epithelial transition factor (c-MET) [12] in HCC cells. miR-34a was reported to participate in HCC by regulating the target genes or signal pathways [13] and also miR-125a-5p played the regulatory role in HCC [14]. However, more researches regarding the definite mechanisms of miR-34a and miR-125a-5p in HCC are demanded.

Metastasis-associated with colon cancer 1 (MACC1), functioning as a colon cancer-related gene and a valid biomarker to predict colon cancer metastasis, targeted 
hepatocyte growth factor (HGF)/MET signaling pathway in colorectal cancer [15]. Afterward, a study reported that MACC1 was identified as a novel prognostic indicator in HCC [16]. Phosphoinositide 3-kinase (PI3K)/Protein Kinase $\mathrm{B}(\mathrm{AKT}) /$ mammalian target of rapamycin (mTOR) is an important pathway modulating cell proliferation, apoptosis, differentiation, and metabolism of various cancers. After activation by receptors, PI3K can transform into phosphatidylinositol (3-5)-triphosphate (PIP3), which recruited AKT to form phosphorylated AKT (p-AKT) that can activate mTOR pathway, affecting downstream gene expression and cellular processes $[17,18]$. PI3K/AKT/mTOR pathway was considered to be implicated in the biological processes of cancers [19-21], including HCC [22]. It has been demonstrated that MACC1 inhibited apoptosis of HCC cells by targeting the PI3K/AKT pathway [17]. Since mTOR can be activated by AKT, MACC1 might have the crucial regulation in the PI3K/AKT/mTOR pathway in HCC.

During this research, the roles of MACC1, miR-34a, and miR-125a-5p in HCC were investigated. What's more, the functional mechanism between miR-34a or miR-125a-5p and MACC1 was explored by further experiments and statistical analysis.

\section{Materials and methods}

Tissues sample and cell culture. 26 pairs of tissues (HCC tissues and matched adjoining normal tissues) were acquired from HCC patients who underwent hepatectomy in Yantaishan Hospital. Acquired tissues were stored in liquid nitrogen for subsequent RNA extraction. Patients didn't receive any treatment for HCC before surgical resection. They were entirely informed and signed written consent. This research got approval from the Ethics Committee of the Yantaishan Hospital.

Human liver epithelial-2 (THLE-2) and human HCC cell line PLC/PRF/5 were acquired from American Type Culture Collection (ATCC, Manassas, VA, USA), and another HCC cell line Huh7 was purchased from the National Type Culture Collection (NTCC, Beijing, China). All cells were cultivated in Dulbecco's modified Eagle's medium (DMEM; Thermo Fisher Scientific, Waltham, MA, USA) complemented with $10 \%$ fetal bovine serum (FBS; GIBCO, Carlsbad, CA, USA) and antibiotics $(100 \mathrm{U} / \mathrm{ml}$ penicillin and $100 \mu \mathrm{g} / \mathrm{ml}$ streptomycin; GIBCO) in a $37^{\circ} \mathrm{C}$ incubator with humid air and $5 \% \mathrm{CO}_{2}$.

Cell transfection. The small interfering RNA (siRNA) targeting MACC1 (si-MACC1\#1 and si-MACC1\#2), short hairpin RNA (shRNA) against MACC1 (sh-MACC1), miR-3 4a mimic (miR-34a), miR-125a-5p mimic (miR-125a-5p), $\mathrm{MACC} 1$ overexpression vector (MACC1), and their negative controls (si-NC, sh-NC, miR-NC, pcDNA) were designed and purchased from RIBOBIO (Guangzhou, China). When cell confluence reached $60 \%$, the mixtures of oligonucleotides/vectors and Lipofectamine 3000 reagent (Thermo
Fisher Scientific) were incubated for $15 \mathrm{~min}$ and instilled to the cells. Transfected cells were prepared for the subsequent assays.

Quantitative real-time polymerase chain reaction (qRT-PCR). To begin with, total RNA was obtained from HCC tissues and cells by Trizol Reagent conforming to the producer's direction. Next, complementary DNA (cDNA) was synthesized using Prime Script ${ }^{\mathrm{TM}} \mathrm{RT}$ reagent kit (Takara, Shiga, Japan) after RNA concentration was determined. Then PCR reaction was conducted with $\mathrm{SYBR}^{\oplus}$ Premix Ex Taq $^{\text {Tw }}$ II Kit (Takara) using ABI Step One Real-time PCR System (Thermo Fisher Scientific). The $2^{-\Delta \Delta C t}$ method was used to calculate and analyze the data, which were standardized by glyceraldehyde-3-phosphate dehydrogenase (GAPDH, for MACC1 mRNA) and U6 (for miRNAs). Primers were synthesized from RIBOBIO: MACC1 (Forward: 5'-CTTGCGGAGGTCACCATAGC-3' and Reverse: 5'-GATTTCCAACAACGGGCTCA-3'); miR-34a (Forward: 5'-CGGTATCATTTGGCAGTGTCT-3' and Reverse: 5'-GTGCAGGGTCCGAGGTAT-3'); miR-125a-5p (Forward: 5'-CGATTCCCTGAGACCCTTTAA-3' and Reverse: 5'-TATGGTTTTGACGACTGTGTGAT-3'); GAPDH (Forward: 5'-GCACCGTCAAGGCTGAGAAC-3' and Reverse: 5'-TGGTGAAGACGCCAGTGGA-3'); U6 (Forward: 5'-ATTGGAACGATACAGAGAAGATT-3' and Reverse: 5'-GGAACGCTTCACGAATTTG-3').

Western blot assay. The extraction of total protein was performed by Radio-Immunoprecipitation Assay (RIPA) lysis buffer with proteinase inhibitor Phenylmethanesulfonyl fluoride (PMSF, Pierce, Rockford, IL, USA) from HCC tissues and cells. Firstly, the quantified proteins were separated through sodium dodecyl sulfate-polyacrylamide gel electrophoresis (SDS-PAGE) for $2 \mathrm{~h}$, followed by transferring onto polyvinylidene fluoride (PVDF) membranes (Thermo Fisher Scientific). Then, 5\% skim milk diluted with phosphate buffered saline (PBS) and 0.1\% Tween 20 (PBST; Thermo Fisher Scientific) was applied to block the membranes for $3 \mathrm{~h}$. After that, the incubation of primary antibodies: anti-MACC1 (Abcam, Cambridge, United Kingdom, ab226803, 1:1000), anti-p-AKT Ser473 (CST, Boston, MA, USA, \#4060, 1:1000), anti-AKT (Abcam, ab81283, 1:1000), anti-p-PI3K Thr458 (CST, \#17366, 1:1000), anti-PI3K (Abcam, ab32089, 1:1000), anti-p-mTOR Ser2448 (CST, \#5536, 1:1000), anti-mTOR (CST, \#2983, 1:1000) and anti- $\beta$-actin (Abcam, ab8227, 1:3000) was administrated overnight at $4^{\circ} \mathrm{C}$. Subsequently, the membranes were washed and incubated with a secondary antibody (Abcam, ab205718, 1:5000) for $1 \mathrm{~h}$ at room temperature. Eventually, the chromogenic reaction was finished by the enhanced chemiluminescence (ECL) reagent (SigmaAldrich, St. Louis, MO, USA) and the results were observed employing ImageJ software (NIH, Bethesda, MD, USA).

Cell Counting Kit-8 (CCK-8). Huh7 and PLC/PRF/5 cells were seeded in a 96-well plate and transfected with plasmids for $0 \mathrm{~h}, 24 \mathrm{~h}, 48 \mathrm{~h}, 72 \mathrm{~h}$. Where after, $10 \mu \mathrm{l} \mathrm{CCK}-8$ reagent (Sigma-Aldrich) was used for incubating cells for $2 \mathrm{~h}$. After 
the dissolution of formazan, the concentration was assessed by the optical density (OD) value at $450 \mathrm{~nm}$ under a microplate reader (Thermo Fisher Scientific).

Transwell migration and invasion assays. In the migration assay, transfected cells were rinsed by PBS and resuspended with a serum-free medium. In the transwell 24-well chamber (Corning Life Sciences, Corning, NY, USA), $600 \mu 1$ DMEM medium with $10 \%$ FBS was added to the lower chamber and resuspended cells were seeded into the upper chamber to incubate for $24 \mathrm{~h}$. Then, the cells were fixed with methyl alcohol and dyed with crystal violet (Sangon, Shanghai, China). Finally, the migrated cells could be observed under a microscope. But in invasion assay, the lower surface of the upper chamber was firstly covered with Matrigel (Corning Life Sciences), and then the rest procedures proceeded as the migration assay above.

Flow cytometry. At $24 \mathrm{~h}$ post-transfection, Huh7 and PLC/PRF/5 cells were digested by $0.25 \%$ pancreatin (GIBCO) without Ethylene Diamine Tetraacetic Acid (EDTA). Harvested cells were rinsed via pre-cooled PBS and resuspended with $1 \times$ binding buffer. Afterward, the Annexin V-fluorescein isothiocyanate (Annexin V-FITC) and propidium iodide (PI) Kit (Sigma-Aldrich) was used to stain cells, complying with the protocol. Apoptotic cells were determined through the flow cytometer (Becton Dickinson, San Jose, CA, USA).

Xenograft tumor assay. The six-week-old $\mathrm{BALB} / \mathrm{c}$ nude mice from Shanghai SIPPR-BK Laboratory Animal Co. Ltd. (Shanghai, China) were subcutaneously injected with Huh-7 cells transfected with sh-MACC1 or sh-NC (6 mice per group). Tumor volume (length $\times$ width $\left.^{2} \times 0.5\right)$ was calculated weekly according to the measurement data. After 5 weeks, tumor tissues were excised from all mice executed euthanasia and weighed. Where after, the expression of MACC1 was detected by western blot. Approved by the Animal Ethics Committee of Yantaishan Hospital, this animal experiment was implemented and abided by the guidelines of Animal Care and Use Committee of the National Institutes of Health.

Dual-luciferase reporter assay. DianaTools database was utilized to predict the targets of miRNA. Briefly, the 3'-UTR sequences of wild-type (WT) MACC1 with the binding sites of miR-34a or miR-125a-5p and 3'-UTR of mutanttype (MUT) MACC1 with the mutated sites were severally cloned into PGL-3 luciferase basic vector (Promega,
Madison, WI, USA), generating luciferase reporter vectors of MACC1\#1 3'-UTR WT, MACC1\#2 3'-UTR WT, MACC1\#1 3'-UTR MUT and MACC1\#2 3'-UTR MUT. HCC cells were co-transfected with MACC1\#1 3'-UTR WT or MACC1\#1 3'-UTR MUT and miR-34a or miR-NC by Lipofectamine 3000 (Thermo Fisher Scientific), along with MACC1\#2 3'-UTR WT or MACC1\#2 3'-UTR MUT and miR-125a-5p or miR-NC. Cells were lysed through $1 \times$ Passive Lysis Buffer (PLB; Promega) at $48 \mathrm{~h}$ post-transfection. Ultimately, the luciferase activities of firefly and internal control renilla were examined by the dual-luciferase reporter system (Promega).

RNA Immunoprecipitation (RIP) assay. RIP assay was implemented via the Magna RIP $^{\mathrm{m}}$ RNA-binding protein immunoprecipitation kit (Sigma-Aldrich) in accordance with the user's manual. In brief, collected cells were incubated for $24 \mathrm{~h}$ using magnetic beads enveloped by anti-Argonaute 2 (anti-Ago2; Abcam, ab32381) with immunoglobulin G (antiIgG; Abcam, ab205718) as a negative control. Subsequently, the magnetic beads were washed and digested with proteinase $\mathrm{K}$ to acquire the protein. RNA was purified applying the phenol-chloroform-isoamyl alcohol reagent (Sangon), and qRT-PCR was conducted to detect the RNA.

Statistical analysis. Collected data were overall presented as the mean \pm standard deviation (SD) of three independent repetitions. SPSS 19.0 and GraphPad Prism 7 were used to carry out statistical analysis. The linear relation between miRNA and MACC1 in HCC tissues was analyzed by Spearman's correlation coefficient. Differences were analyzed through the Student's t-test and one-way analysis of variance (ANOVA) followed by Tukey's test. Statistically, p-value $<0.05$ was recognized as significant.

\section{Results}

MACC1 was upregulated in HCC tissues and cells. A previous study has reported the abnormal overexpression of MACC1 in HCC, and MACC1 was associated with apoptosis of HCC cells [17]. To investigate more biological effects of MACC1 on HCC, we first determined the expression of MACC1 in HCC tissues and cells. Through the analysis of qRT-PCR and western blot, the mRNA and protein levels of MACC1 were dramatically upregulated in HCC tissues in comparison to normal liver tissues (Figures 1A, 1B). Besides, the MACC1 mRNA expression was markedly higher in
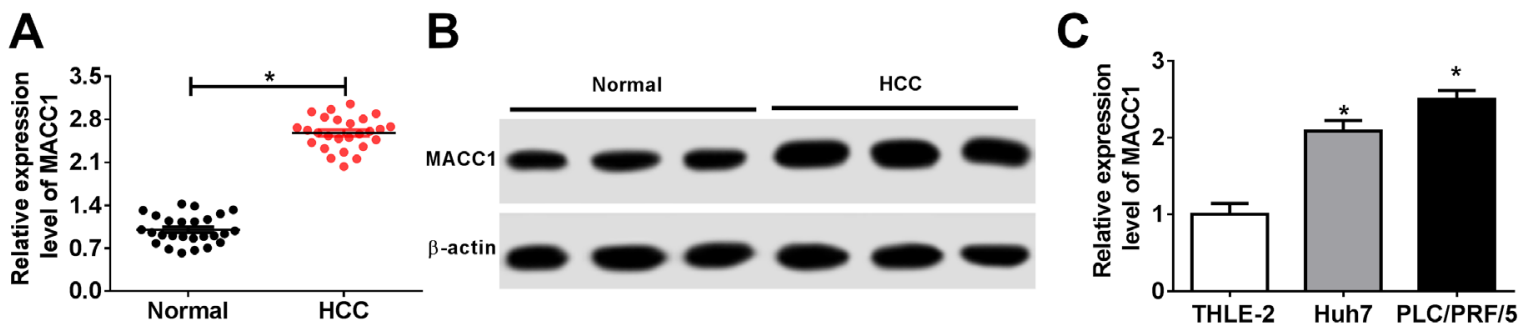

Figure 1. MACC1 was upregulated in HCC tissues and cells. A, B) The expression of MACC1 in HCC and normal tissues was determined by $\mathrm{qRT}$-PCR and western blot. C) MACC1 expression was analyzed by qRT-PCR in HCC cell lines and normal liver cells. ${ }^{*} \mathbf{p}<0.05$ 
Huh7 and PLC/PRF/5 cells than that in normal THLE- 2 cells (Figure 1C). The upregulation of MACC1 in HCC tissues and cells implied that MACC1 might play a crucial role in HCC cellular processes.

The knockdown of MACC1 suppressed proliferation and metastasis but facilitated apoptosis of HCC cells. To explore the role of MACC1 on the cellular processes of HCC, Huh7 and $\mathrm{PLC} / \mathrm{PRF} / 5$ cells were transfected with si-MACC1\#1, si-MACC1\#2 or si-NC. Compared with the si-NC group, a signal decrease of MACC1 mRNA (Figure 2A) and protein (Figure 2B) expression was emerged in the si-MACC1\#1 and si-MACC1\#2 groups, suggesting the great interference effect of si-MACC1\#1 and si-MACC1\#2 in Huh7 and PLC/PRF/5 cells. CCK-8 revealed that the $\mathrm{OD}$ values of different time points at $450 \mathrm{~nm}$ were distinctly lessened in Huh7 (Figure 2C) and PLC/ $\mathrm{PRF} / 5$ (Figure 2D) cells transfected with si-MACC1\#1 or si-MACC1\#2. Next, we measured the cell metastasis ability by transwell migration and invasion assays, in which fewer migrated and invaded cells were in the si-MACC1 groups than that in the si-NC group (Figures 2E, 2F). Besides, flow cytometry reflected the apoptosis rate was significantly boosted by the transfection of si-MACC1\#1 or si-MACC1\#2 (Figure 2G). All these results demonstrated that the knockdown of MACC1 strikingly inhibited cell proliferation and metastasis while promoted apoptosis in HCC cells.

Downregulation of MACC1 decreased tumor growth of HCC in vivo. Xenograft experiment was performed to ascertain the influence of MACC1 in vivo. As shown in Figure 3A, the tumor volume in the sh-MACC1 group was much smaller than that

Figure 2. The knockdown of MACC1 suppressed proliferation and metastasis but facilitated apoptosis of HCC cells. A, B) The qRTPCR and western blot were used to detect the expression level of MACC1 after transfection with si-MACC1\#1, si-MACC1\#2 or si-NC. C, D) The CCK-8 assay was conducted for examining cell proliferation in Huh7 and PLC/PRF/5 cells. E, F) Transwell assay was utilized for the measurement of migrated and invaded cells. G) Flow cytometry was implemented to determine cell apoptosis. ${ }^{*} \mathbf{p}<0.05$ in the sh-NC group. And obviously, the tumor weight was also reduced by the knockdown of MACC1 (Figure 3B). Western blot proved that after MACC1 was knocked down, the protein expression of MACC1 clearly refrained in excised tissues (Figure 3C). Thus, tumor growth was inhibited in vivo following MACC1 downregulation.

\section{A}

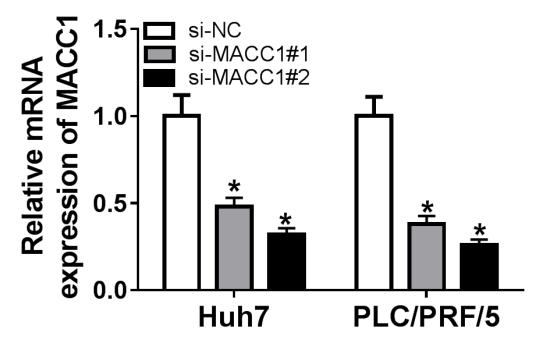

C

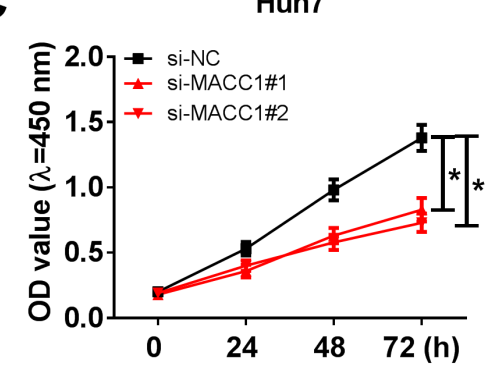

$E$

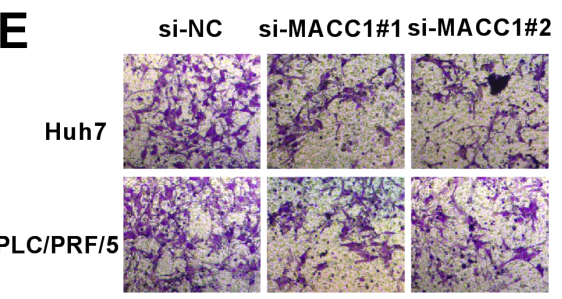

$\mathbf{F}$

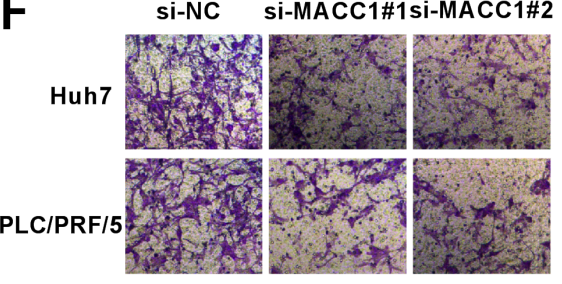

G

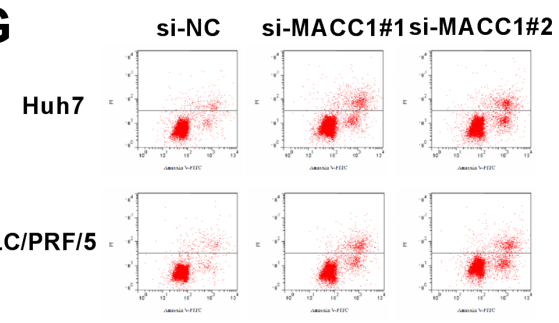

B

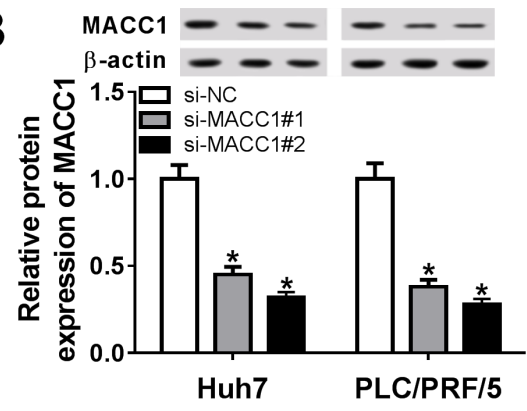

D
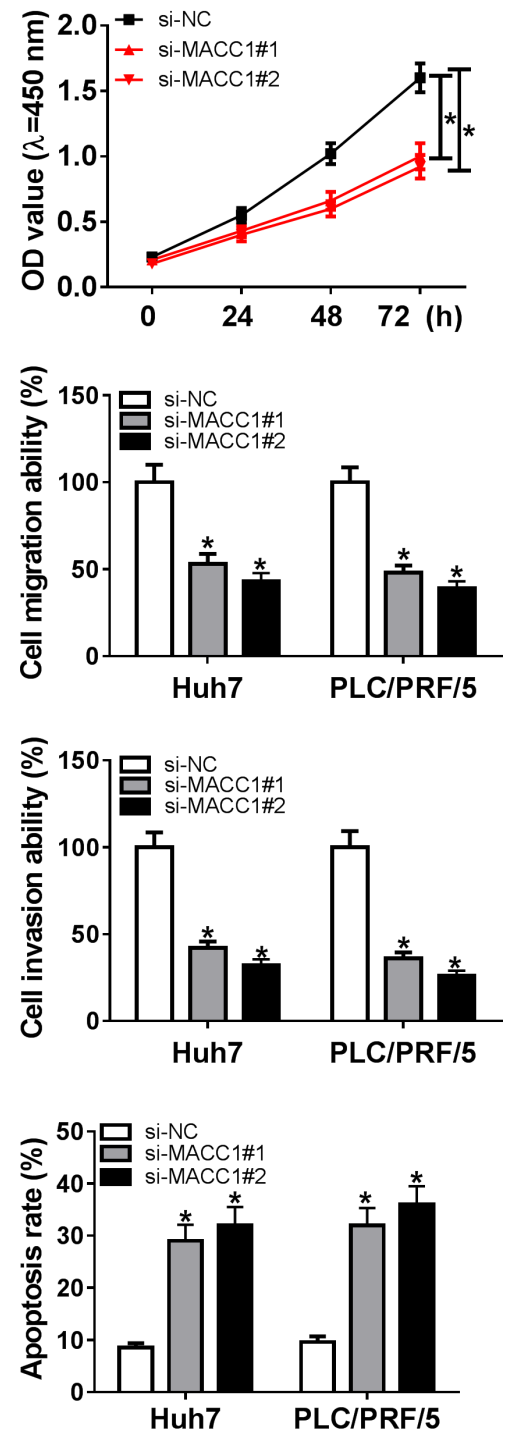

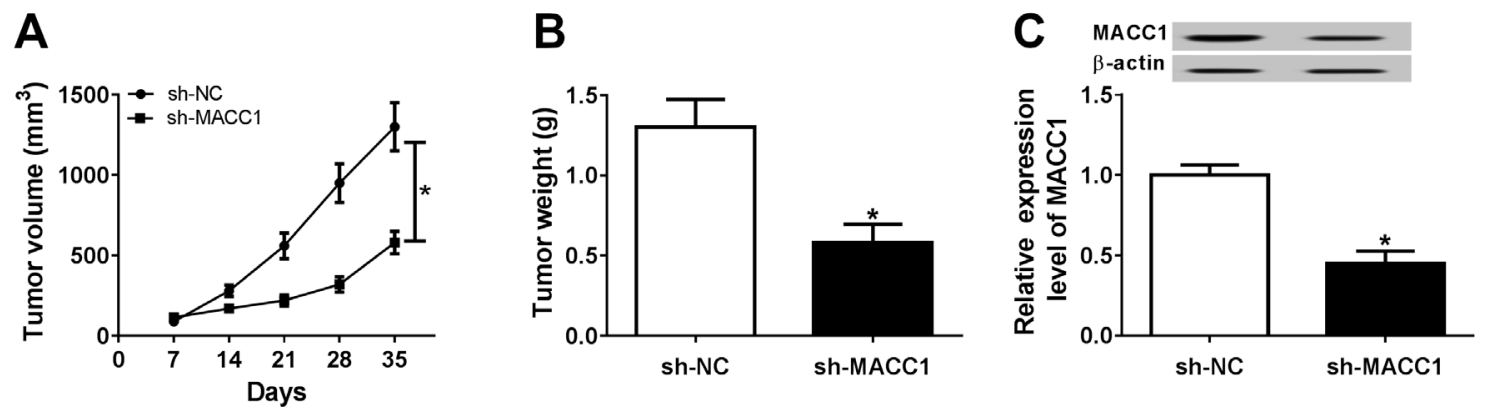

Figure 3. Downregulation of MACC1 decreased tumor growth of HCC in vivo. A) Tumor volume was calculated weekly in sh-NC or sh-MACC1 groups. B) Tumor tissues were excised and weighed. C) The protein expression of MACC1 was measured using western blot. ${ }^{\star}$ p $<0.05$

miR-34a directly targeted MACC1 and suppressed the expression of MACC1 in HCC cells. Predicted by the DianaTools database, MACC1\#1 3'-UTR WT contained the binding sites of miR-34a (Figure 4A). Dual-luciferase reporter assay suggested that the relative luciferase activity of MACC1\#1 3'-UTR WT group was overtly repressed by miR-34a in Huh7 (Figure 4B) and PLC/PRF/5 (Figure 4C) cells, contrasted to MACC1\#13'-UTR MUT group. RIP assay manifested that both MACC1 and miR-34a were enriched in Ago2 pellet by comparison with the IgG NC group, and miR-NC internal control group (Figure 4D). Moreover, the expression of miR-34a was remarkably lower in HCC tissues and cells (Huh7 and PLC/PRF/5) than that in normal tissues and THLE- 2 cells (Figures 4E, 4F). Additionally, a notable negative correlation $(\mathrm{r}=-0.7969, \mathrm{p}<0.0001)$ was observed between the expression of miR-34a and MACC1 in HCC tissues (Figure 4G). And the overexpression efficiency was excellent because of the conspicuous increase of miR-34a level in both Huh7 and PLC/PRF/5 cells transfected with miR-34a (Figure 4H). Western blot assay showed that miR-34a drastically repressed the protein expression of MACC1 in Huh7 and PLC/PRF/5 cells (Figure 4I). The above results testified that $\mathrm{miR}-34 \mathrm{a}$ directly restrained the expression of MACC1 in HCC cells.

Overexpression of $\mathrm{MACC} 1$ reverted the restraint of proliferation and metastasis while enhancement of apoptosis caused by miR-34a in HCC cells. To seek the specific regulatory mechanism of miR-34a and MACC1, Huh7 and PLC/PRF/5 cells were transfected with miR-34a, miR-34a + MACC1 or respective controls. Evidently, MACC1 overexpression alleviated the decreased effect of miR-34a on the protein level of MACC1 (Figure 5A). And transfection of miR-34a overtly reduced cell proliferation, which was abolished by overexpression of MACC1 in Huh7 (Figure 5B) and PLC/PRF/5 (Figure 5C) cells. Also, the decline of cell migration and invasion abilities induced by miR-34a transfection was returned by promoting MACC1 (Figures 5D, 5E). Besides, the transfection of MACC1 manifestly eliminated the miR-34a-induced promoted effect on apoptosis in Huh7 and PLC/PRF/5 cells (Figure $5 \mathrm{~F})$. Taken together, the miR-34a-induced effects on prolif- eration, metastasis, and apoptosis were all reverted by the increase of MACC1.

MACC1 was a direct target of miR-125a-5p. After the prediction of the DianaTools database, we found that MACC1\#2 3'UTR WT had mutual binding points for miR-125a-5p (Figure 6A). And notably, miR-125a-5p reduced the luciferase activity of MACC1\#2 3'UTR WT, whereas this reduction was hindered in the MACC1\#2 3'UTR MUT group (Figures 6B, 6C). Meanwhile, the enrichment of miR-125a-5p and MACC1 was much higher in the Ago2 group than that in IgG and miR-NC groups (Figure 6D). Afterward, we detected the expression level of miR-125a-5p by qRT-PCR, in which miR-125a-5p was downregulated in both HCC tissues (Figure 6E) and cells (Figure 6F) compared to normal tissues and cells. And the relation between miR-125a-5p and MACC1 levels in HCC tissues was markedly negative $(\mathrm{r}=-0.7837, \mathrm{p}<0.0001$, Figure 6G). The qRT-PCR demonstrated that the overexpression effect of miR-125a-5p was conspicuous in both Huh7 and PLC/PRF/5 cells (Figure $6 \mathrm{H}$ ). And there was an apparent decline of MACC1 protein level after the transfection of miR-125a-5p (Figure 6I). Thus, MACC1 was a direct downstream gene of miR-125a-5p.

The upregulation of MACC1 ameliorated the effects of miR-125a-5p on HCC cells. Similarly, the reverted experiment was designed to research the mechanism between miR-125a-5p and MACC1. Through the analysis of western blot, the lessening of MACC1 protein level evoked by miR-125a-5p was relieved following the transfection of MACC1 (Figure 7A). CCK-8 showed that miR-125a-5p led to a striking reduction of cell proliferation in both Huh7 (Figure 7B) and PLC/PRF/5 (Figure 7C) cells, but an ectopic expression of MACC1 alleviated this effect. Likewise, overexpression of MACC1 visually mitigated the inhibition of migration (Figure 7D) and invasion (Figure 7E) but the acceleration of apoptosis (Figure 7F) triggered by miR- $125 \mathrm{a}-5 \mathrm{p}$ in Huh7 and PLC/PRF/5 cells. In short, the effects of miR-125a-5p on HCC cells were all partly abated by MACC1 up-regulation.

miR-34a and miR-125a-5p impeded the PI3K/AKT/ mTOR signaling pathway via repressing MACC1 in HCC cells. Since MACC1 could affect the PI3K/AKT signaling 
A

MACC1\#1 3'UTR WT 5'...UCAAUCCUCUGGUUGCCUGCCA...3'

hsa-miR-34a

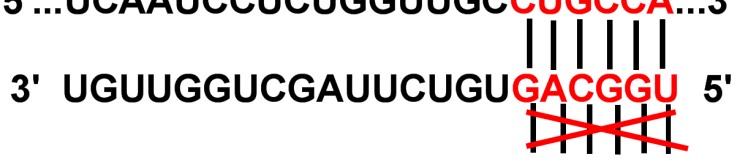

MACC1\#1 3'UTR MUT 5'...UCAAUCCUCUGGUUGCGACGGU... 3'

B

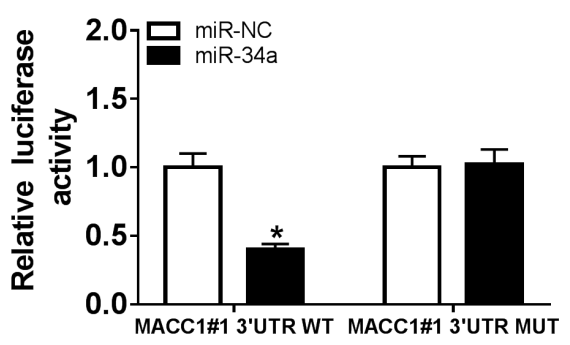

D

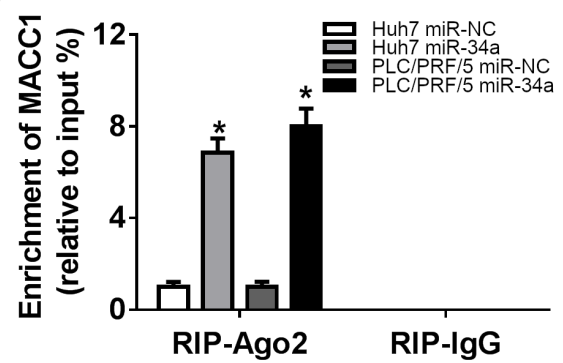

$\mathbf{F}$

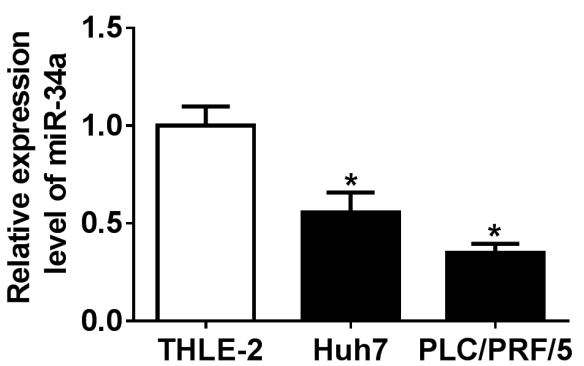

H

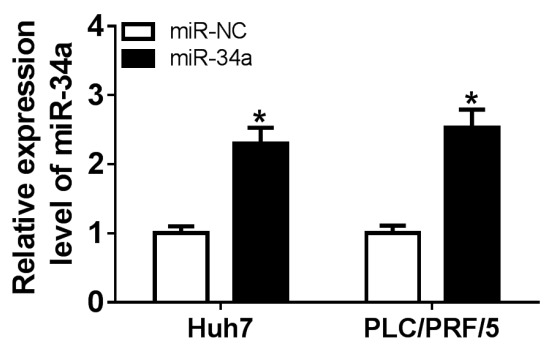

C

PLC/PRF/5

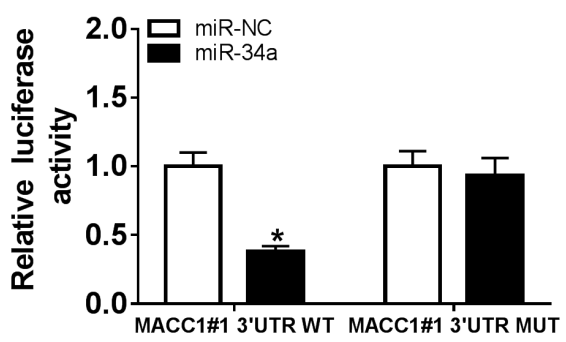

E

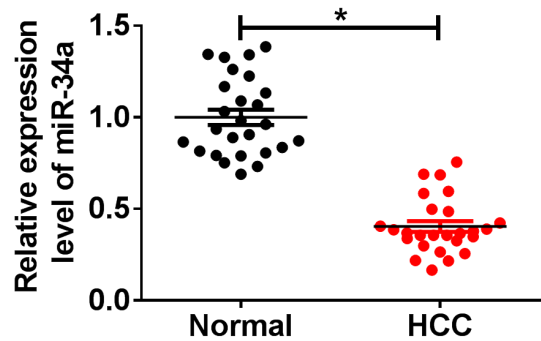

$G$

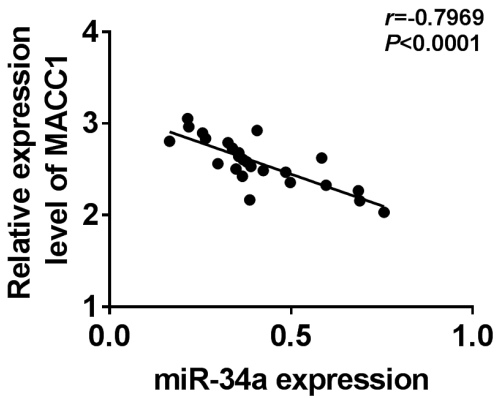

I

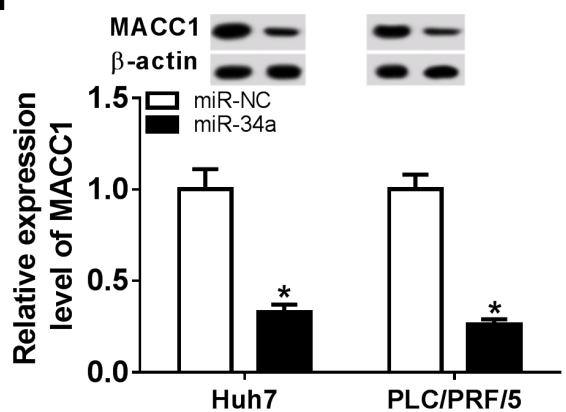

Figure 4. miR-34a directly targeted MACC1 and suppressed the expression of MACC1 in HCC cells. A) Bioinformatics analysis was performed using the DianaTools database. B, C) The relationship between miR-34a and MACC1 in Huh7 and PLC/PRF/5 cells was explored through dual-luciferase reporter assay. D) RIP assay was conducted to verify the relation between miR-34a and MACC1. E, F) The expression level of miR-34a in HCC tissues (E) and cells (F) was detected by qRT-PCR. G) The correlation between miR-34a and MACC1 in HCC tissues was analyzed by Spearman's correlation coefficient. H) Overexpression effect of miR-34a in Huh7 and PLC/PRF/5 cells was measured by qRT-PCR. I) The protein level of MACC1 in Huh7 and $\mathrm{PLC} / \mathrm{PRF} / 5$ cells transfected with miR-34a was examined by western blot. ${ }^{*} \mathrm{p}<0.05$ 


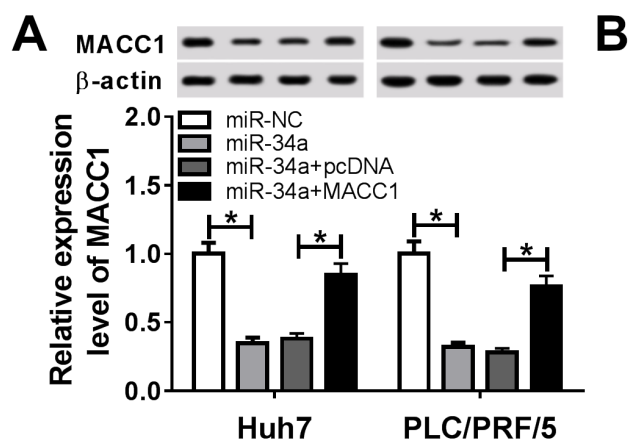

B Huh7

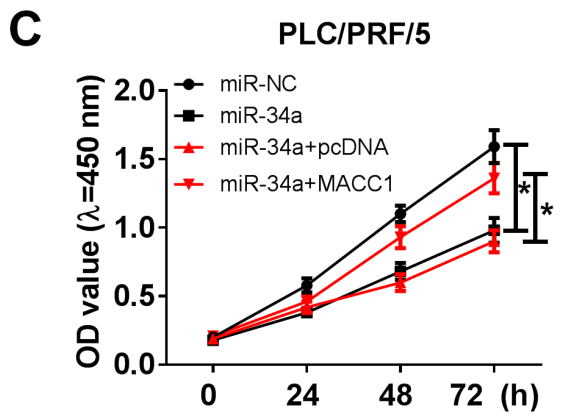

D
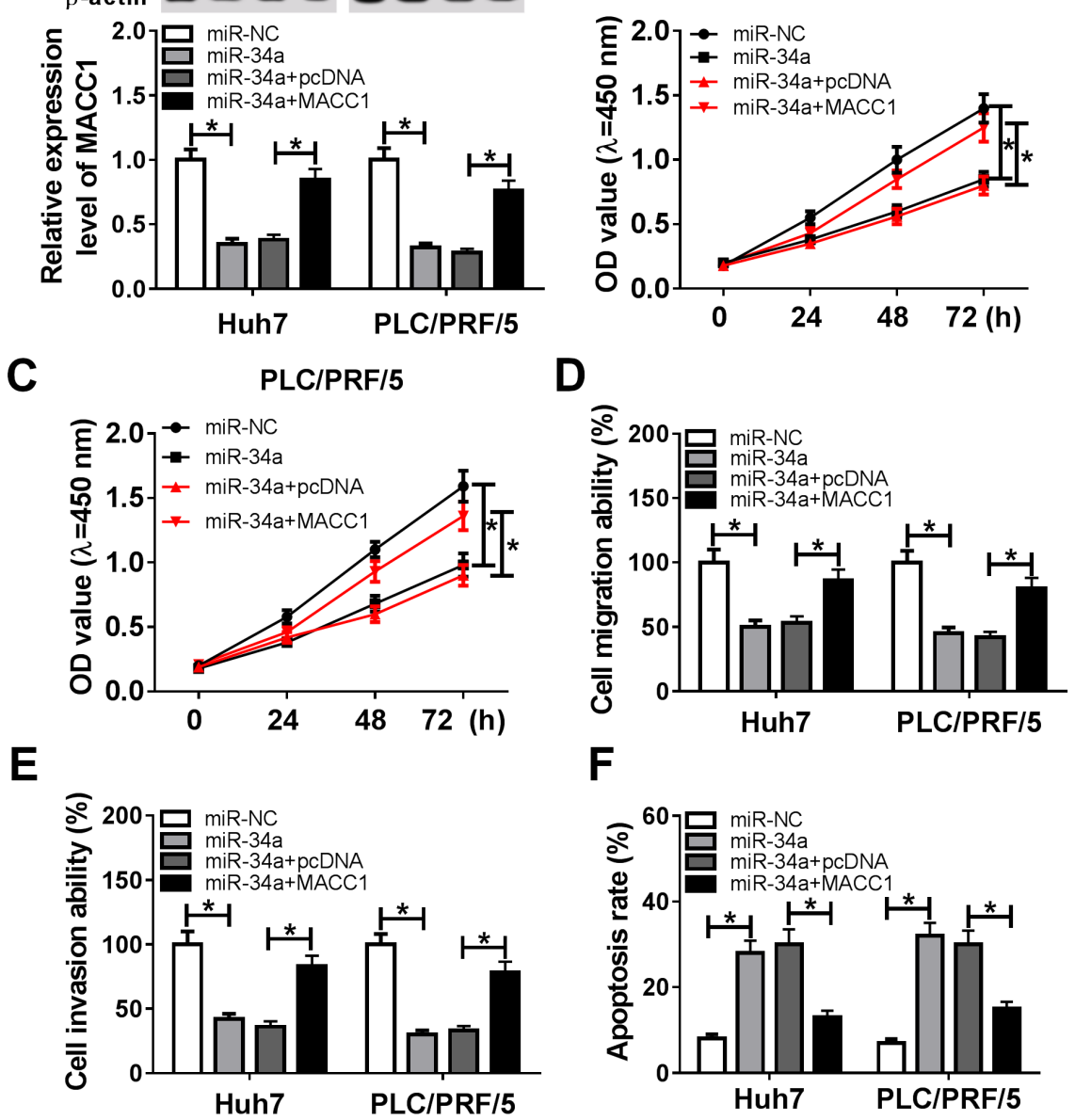

$\mathbf{F}$

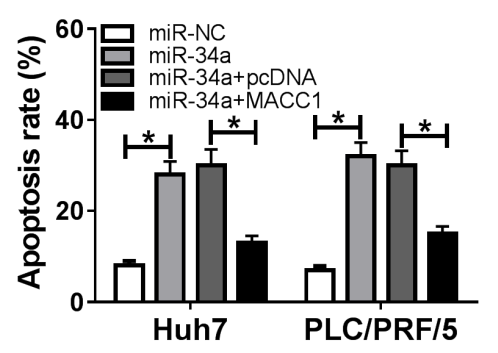

Figure 5. Overexpression of MACC1 reverted the restraint of proliferation and metastasis while the enhancement of apoptosis caused by miR-34a in HCC cells. A) MACC1 protein level was detected by western blot in Huh7 and PLC/PRF/5 cells transfected with miR-34a, miR-34a + MACC1, or relative controls. B, C) Cell proliferation was examined by CCK-8 assay. D, E) Cell migration and invasion were determined by transwell assay. F) Cell apoptosis was measured by flow cytometry. ${ }^{*} \mathrm{p}<0.05$

pathway in HCC cells [17] and it acted as the target of miR-34a or miR-125a-5p through our analysis of results, we used rescue experiments to ascertain whether the effect of MACC1 on the PI3K/AKT signaling pathway was related to miR-34a and miR-125a-5p. After transfection of miR-34a/ miR-125a-5p, miR-34a/miR-125a-5p + MACC1, or their respective negative controls, the protein levels of $\mathrm{p}$-AKT (Ser473), AKT, p-PI3K (Thr458), and PI3K were determined by western blot assay. The levels of p-AKT (Ser473) and p-PI3K (Thr458) were decreased after transfection with miR-34a in Huh7 and PLC/PRF/5 cells, along with the ratios of p-AKT (Ser473)/AKT and p-PI3K (Thr458)/PI3K, whereas the decrease was reversed by MACC 1 overexpression (Figures 8A, 8B). Simultaneously, MACC1 transfection weakened the restrained effect of miR-125a-5p on p-AKT (Ser473)/AKT and p-PI3K (Thr458)/PI3K (Figures 8C, 8D). Additionally, we found that the p-mTOR (Ser2448)/mTOR protein level was refrained by miR-34a or miR-125a-5p overexpression (Supplementary Figure S1), hinting that miR-34a and miR-125a-5p could block the PI3K/AKT/ mTOR pathway via targeting MACC1 in HCC cells.

\section{Discussion}

As one of the most familiar and fatal cancers, HCC brings terrible destruction for the mass of families and a huge threat to public health. The therapeutic strategies still need to improve for relieving the misery and elevating the prognosis outcome of HCC patients. In the last few years, some miRNAs and oncogenes were successively reported to participate in HCC progression [23]. Therefore, it is critical to explore the molecular mechanism between miRNAs and oncogenes in HCC development.

The upregulation of MACC1 was proved to relevant to the lower survival rate in the prognosis of HCC $[16,24]$. In accordance with these reports, the expression of MACC1 


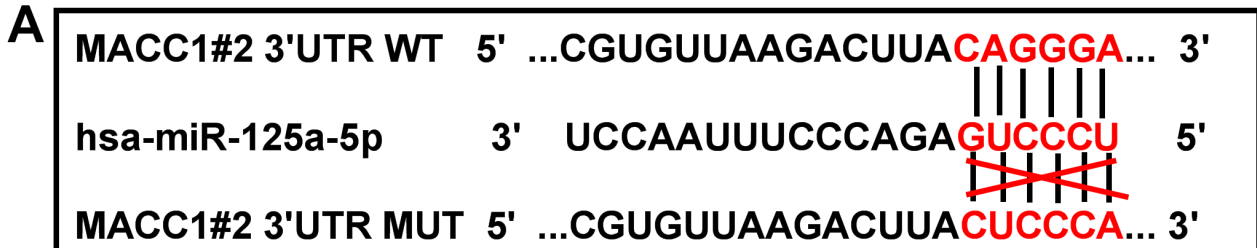

B

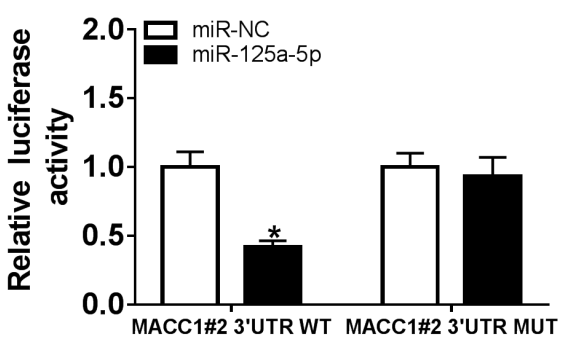

D
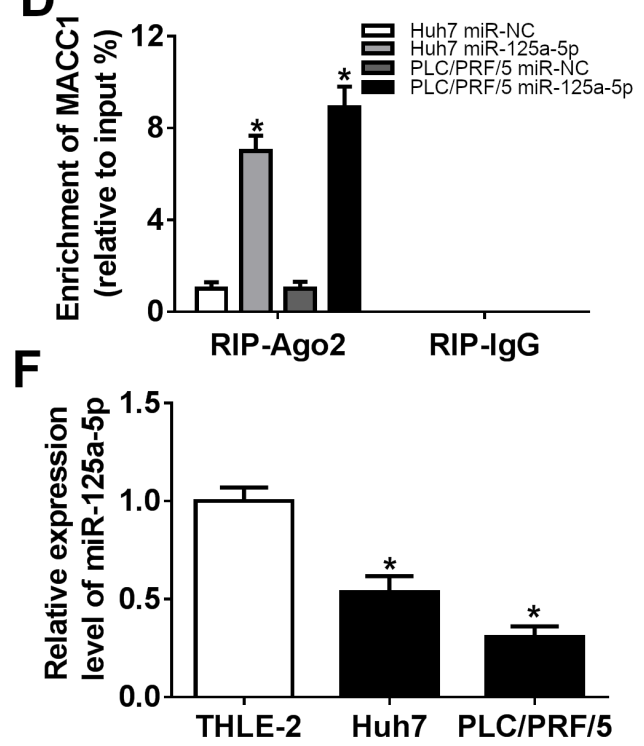

H

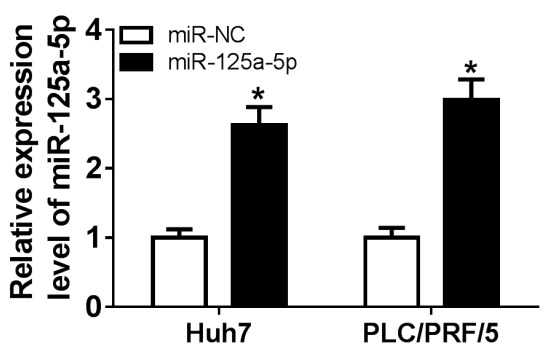

C

PLC/PRF/5

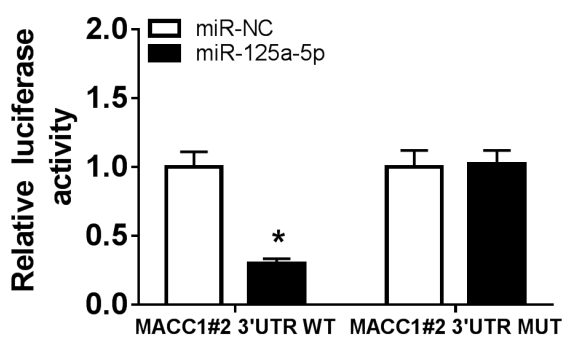

E

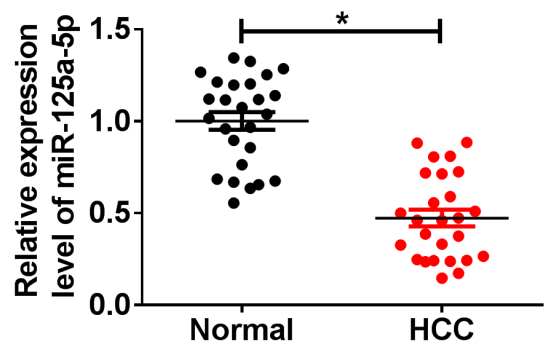

G

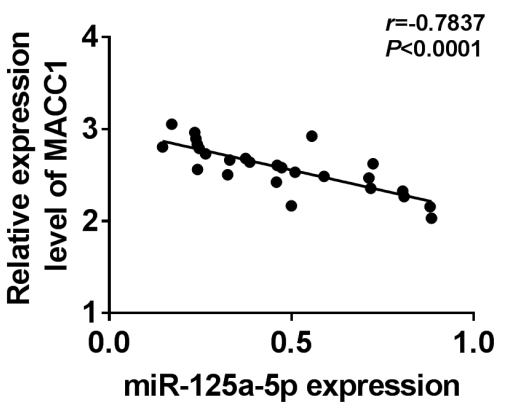

I

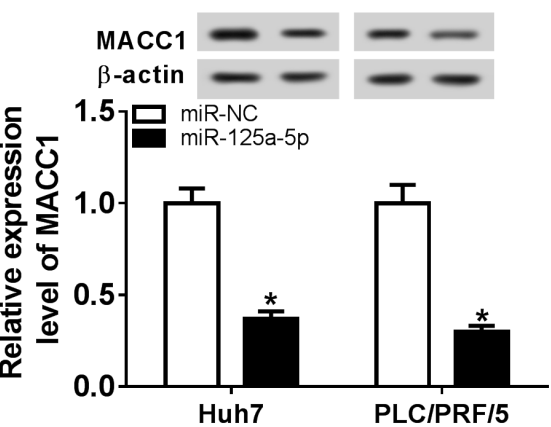

Figure 6. MACC1 was a direct target of miR-125a-5p. A) DianaTools database was applied for predicting the binding sites between miR-125a-5p and MACC1. B-D) The combination between miR-125a-5p and MACC1 was examined by dual-luciferase reporter assay (B, C) and RIP assay (D). E, F) The qRT-PCR was administrated for analyzing miR-125a-5p level in HCC tissues (E) and cells (F). G) Spearman's correlation coefficient was employed for the analysis of the correlation between miR-125a-5p and MACC1 in HCC tissues. H) The qRT-PCR was used for evaluating the overexpression effect of miR-125a-5p in Huh7 and PLC/PRF/5 cells. I) Western blot was implemented to detect the protein level of MACC1 after transfection with miR-125a$5 \mathrm{p} .{ }^{*} \mathrm{p}<0.05$ 

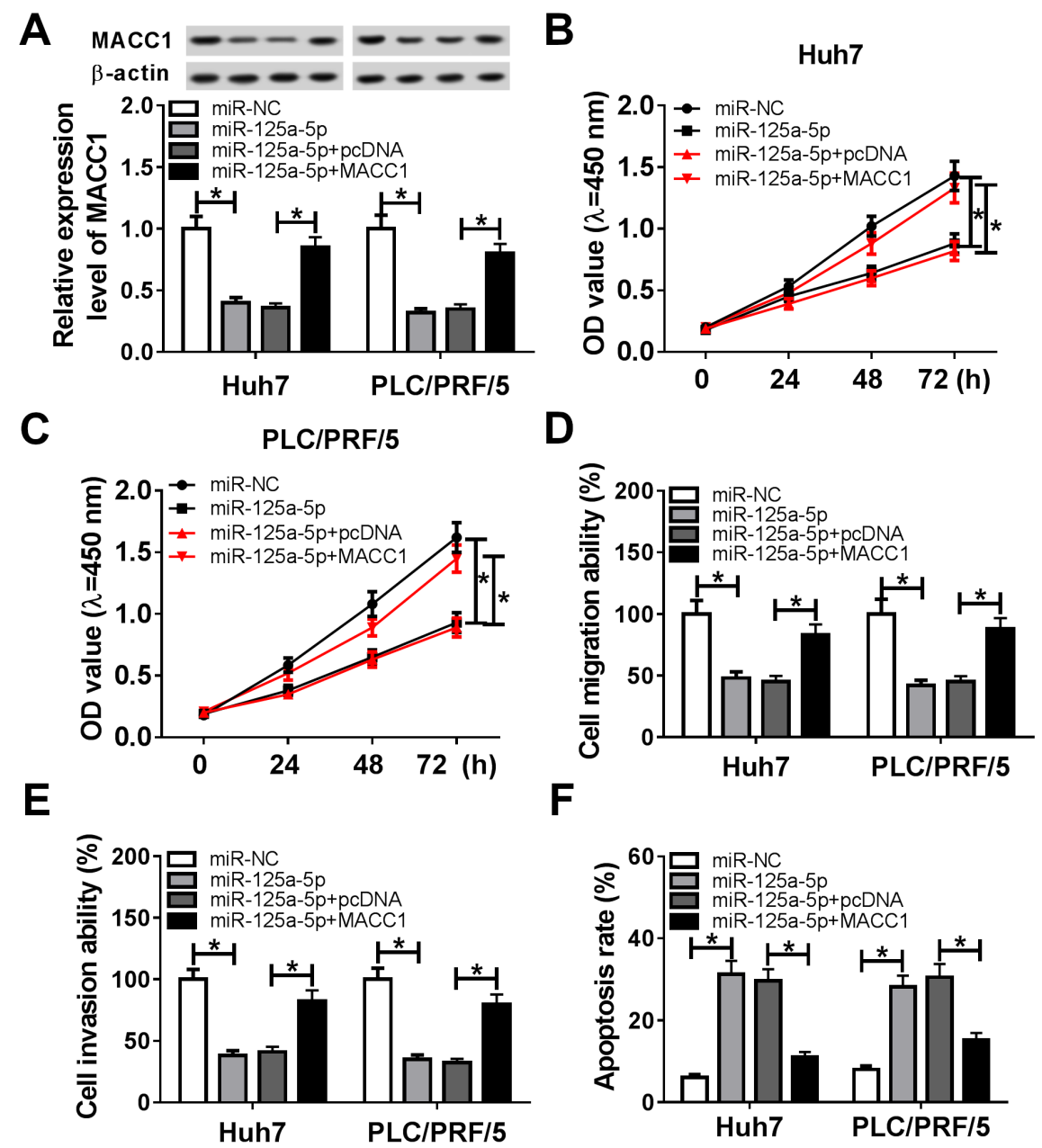

Figure 7. The upregulation of MACC1 ameliorated the effects of miR-125a-5p on HCC cells. A) After transfection with miR-125a-5p, miR-125a-5p + MACC1, or matched controls, western blot was utilized for determining the MACC1 protein level. B, C) The detection of cell proliferation was executed through CCK-8 assay. D, E) The assessment of cell metastasis ability was implemented via transwell migration and invasion assays. F) The examination of apoptotic cells was performed by flow cytometry. ${ }^{*} \mathrm{p}<0.05$

was distinctly boosted in HCC tissues and cells, implicating that MACC1 might play a momentous role in HCC cellular processes. Foregone studies presented that MACC1 resulted in the poor prognosis partially by inducing proliferation [25] and repressing apoptosis via targeting the AKT pathway in HCC [17]. Moreover, Gao et al. found that MACC1 knockdown restrained the metastatic ability of HCC cells [26]. In our study, we attested that the proliferation and metastasis (migration and invasion) were apparently inhibited but the apoptosis was heightened after the knockdown of MACC1 in HCC cells, identical with the above-mentioned studies. Besides, downregulation of MACC1 was presented to inhibit HCC growth in vivo, in conformity with the promotion of HCC growth in vivo by MACC1 overexpression as previously reported [27].

miR-34a was confirmed to regulate the proliferation and apoptosis in different cancer cells $[28,29]$, and miR-125a-5p was also manifested to advance the progression of cancers
[30, 31]. Emerging researches showed that miR-34a was downregulated in HCC [32], then suppressed cell proliferation but induced apoptosis in HCC cells through inhibiting the levels of histone deacetylase 1 (HDAC1) [33] and hexokinase-1(HK-1) [34]. It has also been declared that the miR-125a-5p was expressed at a lower level and refrained tumorigenesis in HCC $[35,36]$. Conformity with the findings, the downregulation of miR-34a and miR-125a-5p was found in this report. And MACC1 was verified as a target of miR-34a and miR-125a-5p. Furthermore, the upregulation of miR-34a and miR-125a-5p both repressed cell proliferation and metastasis while triggered apoptosis by inhibiting MACC1.

MACC1 was reported to be involved in the regulation of glioblastoma via the $\mathrm{PI} 3 \mathrm{~K} / \mathrm{AKT} / \mathrm{mTOR}$ signaling pathway [37]. Interestingly, emerging studies indicated miR-34a and miR-125a-5p improved cancer development by PI3K/ AKT/mTOR pathway. Li et al. asserted that miR-34a had a 

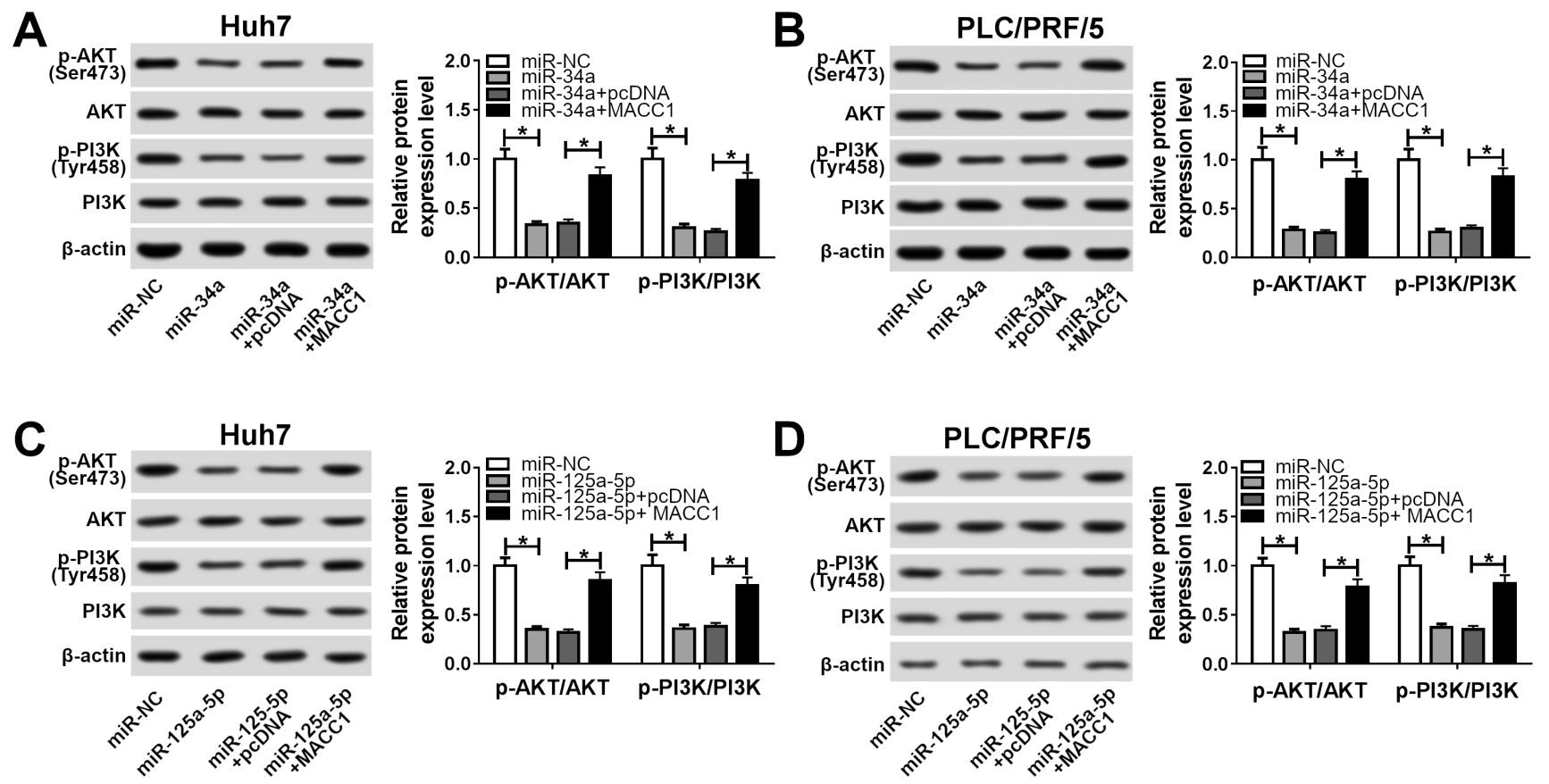

Figure 8. miR-34a and miR-125a-5p impeded the PI3K/AKT signaling pathway via repressing MACC1 in HCC cells. A-D) The protein levels of p-AKT (Ser473), AKT, p-PI3K (Thr458), and PI3K were detected by western blot in Huh7 and PLC/PRF/5 cells transfected with miR-34a, miR-34a + MACC1, or corresponding controls (A, B) and miR-125a-5p, miR-125a-5p + MACC1, or relative controls (C, D). ${ }^{*} \mathrm{p}<0.05$

suppressive influence on the PI3K/AKT/mTOR pathway in colorectal cancer [38]. Besides, Tang et al. proclaimed that miR-125a-5p reduced the invasion capacity of HCC cells by modulating the PI3K/AKT/ mTOR signaling pathway [39]. To confirm whether miR-34a and miR-125a-5p exerted their functions through MACC1 to affect the PI3K/AKT/mTOR pathway, we examined the levels of these pathway-associated proteins. The results suggested that miR-34a or miR-125a-5p inhibited the p-AKT (Ser473)/AKT and p-PI3K (Thr458)/ $\mathrm{PI} 3 \mathrm{~K}$ level by reducing MACC1 and suppressed the expression of p-mTOR (Ser2448)/mTOR, indicating that miR-34a and miR-125a-5p repressed the PI3K/AKT/mTOR signaling pathway in HCC cells via the targeting MACC1.

To sum up, this study expounded that miR-34a and miR-125a-5p refrained proliferation and metastasis while induced apoptosis by suppressing the MACC1-mediated $\mathrm{PI} 3 \mathrm{~K} / \mathrm{AKT} / \mathrm{mTOR}$ pathway in HCC, giving the convincing evidence for the molecular mechanisms of miR-34a and miR-125a-5p in HCC. It is successfully innovative that the regulatory networks of miR-34a/MACC1 or miR-125a-5p/ MACC1 were firstly affirmed in HCC during our report. Prospectively, the miR-34a/miR-125a-5p-MACC1-PI3K/ AKT/mTOR axes might exploit the neoteric vision for the treatment of HCC, affording the firm foundation for the molecular therapies in the clinic. The indicative roles of miR-34a and miR-125a-5p through the regulation of MACC1-PI3K/AKT/mTOR also need to be supported by ample clinical data.
Supplementary information is available in the online version of the paper.

\section{References}

[1] JEMAL A, BRAY F, CENTER MM, FERLAY J, WARD E et al. Global cancer statistics. CA Cancer J Clin 2011; 61: 69-90. https://doi.org/10.3322/caac.20107

[2] CHEN THH, CHEN CJ, YEN MF, LU SN, SUN CA et al. Ultrasound screening and risk factors for death from hepatocellular carcinoma in a high risk group in Taiwan. Int J Cancer 2002; 98: 257-261. https://doi.org/10.1002/ijc.10122

[3] VEL ZQUEZ R. Prospective analysis of risk factors for hepatocellular carcinoma in patients with liver cirrhosis. Hepatology 2003; 37: 520-527. https://doi.org/10.1053/ jhep.2003.50093

[4] EL-SERAG HB, MARRERO JA, RUDOLPH L, REDDY KR. Diagnosis and treatment of hepatocellular carcinoma. Gastroenterology 2008; 134: 1752-1763. https://doi. org/10.1053/j.gastro.2008.02.090

[5] MARTIN RCG, SCOGGINS CR, MCMASTERS KM. Safety and Efficacy of Microwave Ablation of Hepatic Tumors: A Prospective Review of a 5-Year Experience. Ann Surg Oncol 2009; 17: 171-178. https://doi.org/10.1245/s10434-009-0686-Z

[6] CHAPMAN BC, PANICCIA A, HOSOKAWA PW, HENDERSON WG, OVERBEY DM et al. Impact of Facility Type and Surgical Volume on 10-Year Survival in Patients Undergoing Hepatic Resection for Hepatocellular Carcinoma. J Am Coll Surg 2017; 224: 362-372. https://doi.org/10.1016/j. jamcollsurg.2016.11.011 
[7] CHO WC. OncomiRs: the discovery and progress of microRNAs in cancers. Mol Cancer 2007; 6: 60. https://doi. org/10.1186/1476-4598-6-60

[8] GREGORY RI, SHIEKHATTAR R. MicroRNA biogenesis and cancer. Cancer Res 2011; 65: 3509-3512. https://doi. org/10.1158/0008-5472.CAN-05-0298

[9] BUDHU A, JIA HL, FORGUES M, LIU CG, GOLDSTEIN $\mathrm{D}$ et al. Identification of metastasis-related microRNAs in hepatocellular carcinoma. Hepatology 2008; 47: 897-907. https://doi.org/10.1002/hep.22160

[10] DATTA J, KUTAY H, NASSER MW, NUOVO GJ, WANG B et al. Methylation Mediated Silencing of MicroRNA-1 Gene and Its Role in Hepatocellular Carcinogenesis. Cancer Res 2008; 68: 5049-5058. https://doi.org/10.1158/0008-5472. can-07-6655

[11] RASHAD NM, EL-SHAL AS, SHALABY SM, MOHAMED SY. Serum miRNA-27a and miRNA-18b as potential predictive biomarkers of hepatitis $\mathrm{C}$ virus-associated hepatocellular carcinoma. Mol Cell Biochem 2018; 447: 125-136. https://doi.org/10.1007/s11010-018-3298-8

[12] WANG Y, TAI Q, ZHANG J, KANG J, GAO F et al. MiRNA-206 inhibits hepatocellular carcinoma cell proliferation and migration but promotes apoptosis by modulating cMET expression. Acta Biochim Biophys Sin (Shanghai) 2019; 51: 243-253. https://doi.org/10.1093/abbs/gmy119

[13] REN FH, YANG H, HE RQ, LU JN, LIN XG et al. Analysis of microarrays of miR-34a and its identification of prospective target gene signature in hepatocellular carcinoma. BMC Cancer 2018; 18: 12. https://doi.org/10.1186/s12885-0173941-X

[14] KIM JK, NOH JH, JUNG KH, EUN JW, BAE HJ et al. Sirtuin7 oncogenic potential in human hepatocellular carcinoma and its regulation by the tumor suppressors MiR-125a-5p and MiR-125b. Hepatology 2013; 57: 1055-1067. https://doi. org/10.1002/hep.26101

[15] STEIN U, WALTHER W, ARLT F, SCHWABE H, SMITH J et al. MACC1, a newly identified key regulator of HGF-MET signaling, predicts colon cancer metastasis. Nat Med 2009; 15: 59-67. https://doi.org/10.1038/nm.1889

[16] QIU J, HUANGP, QIAN LIU. Identification of maccl as a novel prognostic marker in hepatocellular carcinoma. J Transl Med 2011; 9: 166. https://doi.org/10.1186/1479-58769-166

[17] YAO Y, DOU C, LU Z, ZHENG X, LIU Q. MACC1 suppresses cell apoptosis in hepatocellular carcinoma by targeting the HGF/c-MET/AKT pathway. Cell Physiol Biochem 2015; 35: 983-996. https://doi.org/10.1159/000369754

[18] ZHANG L, WU J, LING M T, ZHAO L, ZHAO K N. The role of the PI3K/Akt/mTOR signalling pathway in human cancers induced by infection with human papillomaviruses. Mol Cancer 2015; 14: 87. https://doi.org/10.1186/s12943-0150361-x

[19] LEAL JFM, LINK W, RENNER O, BLANCO-APARICIO C, CARNERO A. The PTEN/PI3K/AKT Signalling Pathway in Cancer, Therapeutic Implications. Curr Cancer Drug Tar 2008; 8: 187-198. https://doi.org/10.2174/156800908784293659
[20] GHAYAD SE, COHEN PA. Inhibitors of the PI3K/Akt/ mTOR Pathway: New Hope for Breast Cancer Patients. Recent Pat Anticancer Drug Discov 2010; 5: 29-57. https://doi. org/10.2174/157489210789702208

[21] LU C, ZHENGXIANG S, JIANG H, LIXIN Y. MicroRNA92a promotes epithelial-mesenchymal transition through activation of PTEN/PI3K/AKT signaling pathway in nonsmall cell lung cancer metastasis. Int J Oncol 2017; 51: 235244. https://doi.org/10.3892/ijo.2017.3999

[22] WHITTAKER S, MARAIS R, ZHU AX. The role of signaling pathways in the development and treatment of hepatocellular carcinoma. Oncogene 2010; 29: 4989-5005. https://doi. org/10.1038/onc.2010.236

[23] LIN X, CHEN Y. Identification of Potentially Functional CircRNA-miRNA-mRNA Regulatory Network in Hepatocellular Carcinoma by Integrated Microarray Analysis. Med Sci Monit Basic Res 2018; 24: 70-78. https://doi.org/10.12659/ MSMBR.909737

[24] XIE C, WU J, YUN J, LAI J, YUAN Y et al. MACC1 as a Prognostic Biomarker for Early-Stage and AFP-Normal Hepatocellular Carcinoma. Plos One 2013; 8: e64235. 10.1371/ journal.pone.0064235

[25] LI Y, LU Z, LIANG Z, JI D, ZHANG P et al. Metastasis-associated in colon cancer- 1 is associated with poor prognosis in hepatocellular carcinoma, partly by promoting proliferation through enhanced glucose metabolism. Mol Med Rep 2015; 12: 426-434. https://doi.org/10.3892/mmr.2015.3416

[26] GAO J, DING F, LIU Q, YAO Y. Knockdown of MACC1 expression suppressed hepatocellular carcinoma cell migration and invasion and inhibited expression of MMP2 and MMP9. Mol Cell Biochem 2013; 376: 21-32. https://doi.org/10.1007/ s11010-012-1545-y

[27] YAO Y, DOU C, LU Z, ZHENG X, LIU Q. MACC1 suppresses cell apoptosis in hepatocellular carcinoma by targeting the HGF/c-MET/AKT pathway. Cell Physiol Biochem 2015; 35: 983-996. https://doi.org/10.1159/000369754

[28] DENG X, ZHENG H, LI D, XUE Y, WANG Q et al. MicroRNA-34a regulates proliferation and apoptosis of gastric cancer cells by targeting silent information regulator 1 . Exp Ther Med 2018; 15: 3705-3714. https://doi.org/10.3892/ etm. 2018.5920

[29] LIU C, KELNAR K, LIU B, CHEN X, CALHOUN-DAVIS T et al. The microRNA miR-34a inhibits prostate cancer stem cells and metastasis by directly repressing CD44. Nat Med 2011; 17: 211-215. https://doi.org/10.1038/nm.2284

[30] ZHANG Y, ZHANG D, LV J, WANG S, ZHANG Q. MiR125a-5p suppresses bladder cancer progression through targeting FUT4. Biomed Pharmacother 2018; 108: 1039-1047. https://doi.org/10.1016/j.biopha.2018.09.100

[31] LIU H, MA Y, LIU C, LI P, YU T. Reduced miR-125a-5p level in non-small-cell lung cancer is associated with tumour progression. Open Biol 201810; 8: 180118. https://doi. org/10.1098/rsob.180118

[32] WU G, LI Z, WANG Y, JU X, HUANG R. miR-34a Inhibits Cell Proliferation by Targeting SATB2 in Hepatocellular Carcinoma. Biomed Res Int 2018; 2018: 1-7. https://doi. org/10.1155/2018/2863902 
[33] SUN TY, XIE HJ, LI Z, KONG LF, GOU XN et al. miR-34a regulates $\mathrm{HDAC1}$ expression to affect the proliferation and apoptosis of hepatocellular carcinoma. Am J Transl Res 2017; 9: 103-114.

[34] ZHOU Y, LIU K, LIU Y, TAN L. MicroRNA-34a inhibit hepatocellular carcinoma progression by repressing hexokinase-1. J Cell Biochem 2018. https://doi.org/10.1002/ jcb. 27988

[35] XU X, TAO Y, NIU Y, WANG Z, ZHANG C et al. miR-125a$5 p$ inhibits tumorigenesis in hepatocellular carcinoma. Aging (Albany NY) 2019; 11: 7639-7662. https://doi.org/10.18632/ aging.102276

[36] MING M, YING M, LING M. miRNA-125a-5p inhibits hepatocellular carcinoma cell proliferation and induces apoptosis by targeting TP53 regulated inhibitor of apoptosis 1 and Bcl-2-like-2 protein. Exp Ther Med 2019; 18: 1196-1202. https://doi.org/10.3892/etm.2019.7674
[37] ZHOU W, LIU L, XUE Y, ZHENG J, LIU X et al. Combination of Endothelial-Monocyte-Activating Polypeptide-II with Temozolomide Suppress Malignant Biological Behaviors of Human Glioblastoma Stem Cells via miR-590-3p/ MACC1 Inhibiting PI3K/AKT/mTOR Signal Pathway. Front Mol Neurosci 2017; 10: 68. https://doi.org/10.3389/ fnmol.2017.00068

[38] LI Y, ZENG C, HU J, PAN Y, SHAN Y et al. Long noncoding RNA-SNHG7 acts as a target of miR-34a to increase GALNT7 level and regulate PI3K/Akt/mTOR pathway in colorectal cancer progression. J Hematol Oncol 2018; 11: 89. https://doi.org/10.1186/s13045-018-0632-2

[39] TANG H, LI RP, LIANG P, ZHOU YL, WANG GW. miR125a inhibits the migration and invasion of liver cancer cells via suppression of the $\mathrm{PI} 3 \mathrm{~K} / \mathrm{AKT} / \mathrm{mTOR}$ signaling pathway. Oncol Lett 2015; 10: 681-686. https://doi.org/10.3892/ ol.2015.3264 